\title{
ON SOLUTIONS IN THE HYDRODYNAMIC APPROXIMATION OF SOLAR AND STELLAR WINDS WITH VISCOSITY
}

\author{
${ }^{1,2}$ Panagiotis N. Koumantos, ${ }^{2}$ Panaiotis K. Pavlakos and ${ }^{1}$ Xenophon D. Moussas \\ ${ }^{1}$ Department of Physics, National and Kapodistrian University of Athens, \\ Panepistimiopolis GR-15783 Athens, Greece \\ ${ }^{2}$ Department of Mathematics, National and Kapodistrian University of Athens, \\ Panepistimiopolis GR-15784 Athens, Greece
}

Received 2014-05-04; Revised 2014-05-28; Accepted 2014-05-30

\begin{abstract}
In this article we present some results in existence and uniqueness of strong and classical solutions of the hydrodynamic equations modeling solar and stellar winds. The system of Navier-Stokes equations for solar and stellar winds is considered in its corresponding differential evolution equation form $(d / d t+A) v(t)=$ $F(v(t), t)$, where $F$ is a given non-linear function and -A is the infinitesimal generator of the analytic semigroup arising from the hydrodynamic Stokes operator.
\end{abstract}

Keywords: Solar and Stellar Winds, Hydrodynamics, Navier-Stokes Equations, Evolution Equations

\section{INTRODUCTION}

The solar corona, i.e., the outer atmosphere of the Sun, is in continuous hydrodynamical expansion, producing out through the interplanetary medium a flow of plasma, which is known as the solar wind.

Parker (1958) was the first who observed that a fluid description is appropriate and hydrodynamic streaming is possible, at least out to distances at the order of astronomical units from the Sun.

The fully ionized gas of the solar corona, containing approximately $90 \%$ hydrogen and $10 \%$ helium is plasma, described by Boltzmann equations concerning distribution functions for electrons, protons and alphaparticles. Let $N$ the total number density. Let also that all species have the same local temperature $T$ and diffusion effects can be neglected, i.e., they have the same streaming velocity $u$. If $\mathrm{g}$ is the acceleration field, $K$ is the coefficient of thermal conductivity, $k$ is the Boltzmann constant, $\mathrm{m}$ is the mean mass per particle, then for a fluid with isotropic pressure, negligible viscous loss and no external heat source the hydrodynamic equations are:

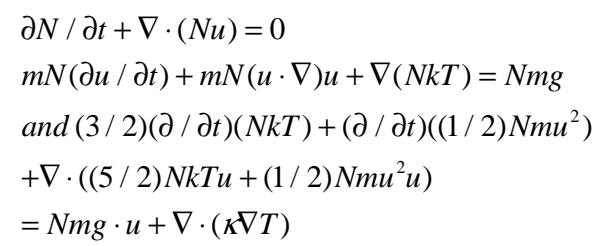

The above equations are the Navier-Stokes equations for motion of a fluid with mass density $\mathrm{Nm}$ and a specific heat ratio of $\gamma=5 / 3$.

Numerical solutions for this equations, as and for the corresponding case including viscosity, have been studied by Noble and Scarf (1963), Summers (1980a) and Whang et al., (1965) describing the streaming solar atmosphere.

The heat capacity ratio $\gamma$ is related straightforwardly to the degrees of freedom of the particles which constitute the specific studied population. Under the study of electron populations of the Solar Wind plasma and for a time-span of many years, by the application of the framework of the Tsallis entropy, that is by the use of kappa distributions, the number of the degrees of freedom of the electron populations of electrons, which 
contribute to the internal energy of the populations, is observed to vary within the passage of time and this variation follows the characteristic periodicities of the Solar Wind. These degrees of freedom may vary from three, corresponding to the motion of the plasma electrons and ions in space, to two, in the case of an axial symmetry, or to one, in the case of spherical symmetry. This has as a consequence that the heat capacity ratio $\gamma$ obtains the value $\gamma=5 / 3$, for $n=3$ degrees of freedom, the value $\gamma=2$, for $n=2$ degrees of freedom and the value $\gamma=3$, for $n=1$ degrees of freedom, accordingly (Gkountras et al. (2014) work in progress).

As has been noted by Summers (1980b) the role of viscosity in solar wind is not well-understood, since the model studied by Scarf and Noble (1965) in which included viscous effects obtained physically unacceptable results, while a well-behaved model including viscosity studied by Whang and Chang (1965) imposed the possibility of physically realistic boundary conditions.

On the other hand Leer and Holzer (1972) studied kinetic and hydrodynamic models of collisionless solar wind proton gas and found that whilst heat conduction and viscosity are neglected in hydrodynamic model, they are automatically included in the kinetic model and the results of the two approximations are nearly the same. Also Leer and Holzer (1972) proposed that models of the quiet solar wind should be based on a hydrodynamic formulation.

For a modern excursion in solar wind and cosmic-ray astrophysics we refer to the book of Schlickeiser (2002).

In this note we establish analytic approximation of the problem and we consider the equations for a hydrodynamic model of solar and stellar winds in the dimensional analysis form:

$$
(\partial u / \partial t)-\Delta u+(u, g r a d) u+\operatorname{grad} p=G
$$

With $\operatorname{div} u=0$ in $X \times R$ and $u \mid \partial X=0$, where $X$ is a smooth domain of $\mathrm{R}^{3}$ and by definition the operator $(u, \operatorname{grad}):=\sum_{j=1}^{3} u_{j}\left(\partial / \partial x_{j}\right)$.

This system describes the motion of viscous incompressible fluid. The function $\mathrm{u}: \mathrm{R}^{3} \times \mathrm{R} \rightarrow \mathrm{R}^{3}$, with $u(x, t)$ $=\left(u_{1}(x, t), u_{2}(x, t), u_{3}(x, t)\right)$ represents the velocity of the fluid, $\mathrm{p}(\mathrm{x}, \mathrm{t})$ is the pressure and $\mathrm{G}: \mathrm{R}^{3} \times \mathrm{R} \rightarrow \mathrm{R}^{3}$ is the given external force, with $G(x, t)=\left(G_{1}(x, t), G_{2}(x, t), G_{3}(x, t)\right)$.

\section{MAIN RESULTS}

In what follows for classical notions and terminology in functional analysis and semigroups we refer to the book of Yosida (1980).
For our approximation we consider the Hilbert space $H$ with elements $u \in L^{2}\left(X, \quad B(X), \quad \lambda^{3}\right)$ such that $\int_{X} u \cdot \nabla \psi(x) d \lambda^{3}(x)=0$ for all $\psi \in W^{1,2}\left(X, B(X), \lambda^{3}\right)$.

By $W^{m, p}\left(X, B(X), \lambda^{3}\right), 1 \leq p<\infty$ as usual we denote the corresponding Sobolev spaces, i.e., the space of all functions $g$ in $L^{p}\left(X, B(X), \lambda^{3}\right)$ such that the distributional derivative $\partial^{s} g \in L^{p}\left(X, B(X), \lambda^{3}\right)$, for all $s \in \mathbf{N}_{0}^{3}$ with $|s| \leq m$ under the norm $\|g\|_{m, p}:=\left(\sum_{0 \leq|s| \leq m} \int_{X}\left|\partial^{s} g(x)\right|^{p} d \lambda^{3}(x)\right)^{\frac{1}{p}}$.

Let the non-negative and self-adjoint Stokes operator $\mathrm{S}$ with domain.

$$
D(S)=W^{2,2}\left(X, B(X), \lambda^{3}\right) \cap W_{0}^{1,2}\left(X, B(X), \lambda^{3}\right) \text { and range }
$$
$R(S)=L^{2}\left(X, B(X), \lambda^{3}\right)$ defined by the formula $S:=-P_{2} \Delta$, where $P_{2}$ denotes the projection operator from $L^{2}(X, B(X)$, $\lambda^{3}$ ) onto $H$ and $\Delta$ is the Laplace operator. Let also $\left\{e^{-t S}: t \geq 0\right\}$ be the analytic semigroup generated by $S$.

Then applying the projection operator $\mathrm{P}_{2}$ to both sides of the Navier-Stokes Equation 1.1 yields:

$$
(\partial / \partial t+S+\mathrm{B}) u=P_{2} G, t \in R
$$

where, $B:=P_{2}(u$, grad) (Giga, 1983; von Wahl, 1985).

Therefore, setting $v: R \rightarrow R^{3}: t \rightarrow v(t):=u(x, t)$, the system of equations can be written as:

$(d / d t+A) v(t)=F(v(t), t), t \in R$

where, the operator $A$ : $=S$ is the non-negative and selfadjoint stokes operator and the function $F$ absorbs the non-linear terms.

In order to deal with the last equation we first consider the corresponding linear evolution equation:

$(d / d t+A) v(t)=f(t), t \in R$

As is well-known, we may assume that there exist constants $\mu_{0}, \delta>0$ such that $\left\|e^{-t A}\right\| \leq \mu_{0} e^{-\delta t}$, for all $t \in R^{+}$.

We denote by $C_{b}\left(R, R^{3}\right)$ the Banach space of bounded continuous functions $\varphi: R \rightarrow R^{3}$, endowed with supremum norm $|\phi|:=\sup \{\|\phi(t)\|: t \in R\}$ and let $C\left(R, R^{3}\right)$ the space of continuous functions $\varphi: R \rightarrow R^{3}$. Also by $P\left(R, R^{3}\right)$ we denote the closed subspace of $C_{b}\left(R, R^{3}\right)$ of all almost periodic functions in $C_{b}\left(R, R^{3}\right)$.

Let also $M^{1}\left(R, R^{3}\right)$ be the Banach space of Bochnermeasurable functions $\varphi: \quad R \rightarrow R^{3}$ for which $\int_{t}^{t+1}\|\phi(s)\| d \lambda^{1}(s)<+\infty$, for all $t \in R$, under the norm $|\phi|_{M^{1}}:=\sup \left\{\int_{t}^{t+1}\|\phi(s)\| d \lambda^{1}(s): t \in \mathrm{R}\right\}$. 
A function $\varphi: R \rightarrow \operatorname{Dom}(A)$ is called a strong solution (respectively a classical solution) on $R$ of (2.1) or (2.2) if it is strongly differentiable for $\lambda^{1}$-almost every $t \in R$ (respectively for every $t \in R$ ).

In the sense of semigroup approach to differential evolution equations, concerning the case of magnetohydodynamics (Athanasiadou et al., 2014a; $2014 \mathrm{~b}$ ), for the hydrodynamic approximation that we are intrested in, we obtain the following results.

\section{Proposition 2.1}

If $f \in M^{1}\left(\mathrm{R}, \mathrm{R}^{3}\right)$ then Equation 2.2 has at least one strong solution $v$ in $C_{b}\left(R, R^{3}\right)$.

Proof. Let $v: R \rightarrow R^{3} t \rightarrow v(t):=\int_{-\infty}^{t} e^{-(t-s) A} f(s) d \lambda^{1}(s)$.

Then, for every $t \in R$ :

$$
\begin{aligned}
& \int_{-\infty}^{t}\left\|e^{-(t-s) A} f(s)\right\| d \lambda^{1}(s) \\
& \leq \mu_{0} \int_{0}^{\infty} e^{-\delta s}\|f(t-s)\| d \lambda^{1}(s) \\
& \leq \mu_{0} \sum_{n=0}^{\infty} \int_{n}^{n+1} e^{-\delta s}\|f(t-s)\| d \lambda^{1}(s) \\
& \leq \mu_{0}\left(\sum_{n=0}^{\infty} e^{-\delta n}\right)|f|_{M^{1}} \leq \frac{\mu_{0}}{1-e^{-\delta}}|f|_{M^{1}}
\end{aligned}
$$

Hence, $v \in C_{b}\left(R, R^{3}\right)$.

For $h>0$ follows:

$$
\begin{aligned}
& h^{-1}(v(t+h)-v(t)) \\
& =h^{-1}\left(e^{-h A}-I\right) \int_{-\infty}^{t} e^{-(t-s) A} f(s) d \lambda^{1}(s) \\
& +h^{-1} \int_{t}^{t+h} e^{-(t+h-s) A}(f(s)-f(t)) d \lambda^{1}(s) \\
& +h^{-1} \int_{t}^{t+h}\left(e^{-(t+h-s) A}-I\right) f(t) d \lambda^{1}(s)+f(t)
\end{aligned}
$$

Letting $\quad h \rightarrow 0+\quad$ we conclude $\dot{v}(t)=\lim _{h \rightarrow 0+}(v(t+h)-v(t))=-A v(t)+f(t)$, for almost every $t \in R$, i.e., $v$ is a strong solution in $C_{b}\left(R, R^{3}\right)$.

Let $\Phi$ the corresponding Nemytskii operator of the non-linear function $F: R^{3} \times R \rightarrow R^{3}$ appearing in Equation 2.1 , i.e., for every $y: R \rightarrow R^{3}, \Phi$ y is defined by $\Phi y(t):=$ $F(y(t), t), t \in R$.

\section{Proposition 2.2}

Let $\Phi y \in M^{1}\left(R, R^{3}\right)$ provided $y \in M^{1}\left(R, R^{3}\right)$ and there exists a constant $\eta>0$ such that $\left\|\Phi y_{1}-\Phi y_{2}\right\| \leq \eta\left\|y_{1}-y_{2}\right\|$ for all $y_{1}, y_{2} \in M^{1}\left(R, R^{3}\right)$ and $\mu_{0} \delta^{-1} \eta<1$. Then Equation 2.1 has at least one strong solution $v$ in $M^{1}\left(R, R^{3}\right)$.
Proof. We consider the Hamerstein-type operator $\Xi: M^{1}\left(\mathrm{R}, \mathrm{R}^{3}\right) \rightarrow M^{1}\left(\mathrm{R}, \mathrm{R}^{3}\right)$ which to any $y \in M^{1}\left(R, R^{3}\right)$ associates a strong solution $\Xi \mathrm{y}$ in $C_{b}\left(R, R^{3}\right) \subseteq M^{1}\left(R, R^{3}\right)$ of the linear evolution equation $\dot{x}(t)+A x(t)=\Phi y(t)$ such that $\Xi y=\int_{-\infty}^{t} e^{-(t-s) A} \Phi y d \lambda^{1}(s), t \in R$. Let $t, \sigma \in R$ and $y_{l}$, $y_{2} \in M^{1}\left(R, R^{3}\right)$. Then combining the assumptions and Fubini theorem it follows:

$$
\begin{aligned}
& \int_{\sigma}^{\sigma+1}\left\|\Xi y_{1}(t)-\Xi y_{2}(t)\right\| d \lambda^{1}(s) \\
& \leq \int_{\sigma}^{\sigma+1} \int_{0}^{\infty}\left\|e^{-s A}\right\|\left\|\Phi y_{1}(t-s)-\Phi y_{2}(t-s)\right\| d \lambda^{1}(s) d \lambda^{1}(t) \\
& \leq \mu_{0} \delta^{-1} \eta\left|y_{1}-y_{2}\right|_{M^{1}}
\end{aligned}
$$

Thus, $\Xi$ is a contraction operator in $M^{1}\left(R, R^{3}\right)$. By similar arguments we have the next result.

\section{Proposition 2.3}

Let $\Phi y \in P\left(R, R^{3}\right)$ provided $y \in P\left(R, R^{3}\right)$ and there exists a constant $\eta>0$ such that $\left\|\Phi y_{1}-\Phi y_{2}\right\| \leq \eta\left\|y_{1}-y_{2}\right\|$ for all $y_{1}, y_{2} \in P\left(R, R^{3}\right)$ with $\mu_{0} \delta^{-1} \quad \eta<1$. Then there exists exactly one classical solution $v$ of Equation 2.1 in $P\left(R, R^{3}\right)$.

\section{REFERENCES}

Athanasiadou, E.S., V.F. Dionysatos, P.N. Koumantos and P.K. Pavlakos, 2014a. A semigroup approach to functional differential evolution equations. Math. Meth. Appl. Sci., 37: 217-222. DOI: 10.1002/mma.2891

Athanasiadou, E.S., V.F. Dionysatos, P.N. Koumantos and P.K. Pavlakos, 2014b. Almost Periodic Solutions of Navier-Stokes-Ohm Type Equations in Mag-Netohydrodynamics, Applications of Mathematics and Informatics to Science and Engineering. In: Springer Optimization and Its Applications, Daras, N.J. (Ed.), ISBN-10: 3319047191, pp: 43-57.

Giga, Y., 1983. Weak and strong solutions of the Navier- Stokes initial value problem. Publ. RIMS, Kyoto Univ. 19: 887-910. DOI: 10.2977/prims/1195182014

Gkountras, T., X. Moussas and P. Papaspirou, 2014. Work in progress.

Leer, E. and T.E. Holzer, 1972. Collisionless solar wind protons: A comparison of kinetic and hydrodynamic descriptions. J. Geophys. Res., 77: 4035-4041. DOI: 10.1029/JA077i022p04035 
Noble, L.M. and F.L. Scarf, 1963. Conductive heating of the solar wind I. Astrophys. J., 138: 1169-1181. DOI: $10.1086 / 147715$

Parker, E.N., 1958. Dynamics of the interplanetary gas and magnetic fields. Astrophys. J., 128: 664-676. DOI: $10.1086 / 146579$

Scarf, F.L. and L.M. Noble, 1963. Conductive heating of the solar wind II. The inner corona, Astrophys. J., 141: 1479-1491. DOI: 10.1086/148236

Schlickeiser, R., 2002. Cosmic Ray Astrophysics. 2nd Ed., Springer, Berlin, ISBN-10: 3540664653, pp: 519.

Summers, D., 1980a. Spurious solutions of the NavierStokes equations. Astrophys. J., 241: 468-473. DOI: $10.1086 / 158360$

Summers, D., 1980b. Stellar wind theory. J. Geophys. Rec., 85: 2277-2284. DOI: 10.1029/JA085iA05p02277 von Wahl, W., 1985. The Equations of Navier-Stokes and Abstract Parabolic Equations. 1st Ed., Vieweg, Braunschweig, ISBN-10: 3528089156, pp: 264.

Whang, Y.C., C.K. Liu and C.C. Chang, 1965. A viscous model of the solar wind. Astrophys. J., 145: 255269. DOI: $10.1086 / 148760$

Whang, Y.C. and C.C. Chang, 1965. An inviscid model of the solar wind, J. Geophys. Res., 70: 1475-4180. DOI: 10.1029/JZ070i017p04175

Yosida, K., 1980. Functional Analysis. 6th Ed. SpringerVerlag Berlin Heidelberg New York, ISBN: 3-45058654-7. 